\title{
The PML nuclear bodies-associated protein TTRAP regulates ribosome biogenesis in nucleolar cavities upon proteasome inhibition
}

\author{
S Vilotti ${ }^{1}$, M Biagioli ${ }^{1,2}$, R Foti ${ }^{1}$, M Dal Ferro ${ }^{3,4}$, Z Scotto Lavina ${ }^{1,2}$, L Collavin ${ }^{3,4}$, G Del Sal ${ }^{3,4}$, S Zucchelli, ${ }^{\star 1,2,5}$ and S Gustincich ${ }^{\star, 1,2,5}$ \\ TRAF and TNF receptor-associated protein (TTRAP) is a multifunctional protein that can act in the nucleus as a $5^{\prime}$-tyrosyl DNA \\ phosphodiesterase and in the cytoplasm as a regulator of cell signaling. In this paper we show that in response to proteasome \\ inhibition TTRAP accumulates in nucleolar cavities in a promyelocytic leukemia protein-dependent manner. In the nucleolus, \\ TTRAP contributes to control levels of ribosomal RNA precursor and processing intermediates, and this phenotype is \\ independent from its $5^{\prime}$-tyrosyl DNA phosphodiesterase activity. Our findings suggest a previously unidentified function for \\ TTRAP and nucleolar cavities in ribosome biogenesis under stress.
}

Cell Death and Differentiation (2012) 19, 488-500; doi:10.1038/cdd.2011.118; published online 16 September 2011

TRAF and TNF receptor-associated protein (TTRAP) is a 5 'tyrosyl DNA phosphodiesterase that is required for the repair of topoisomerase2-induced DNA double-strand breaks. ${ }^{1}$ It is a member of the endonuclease/exonuclease/ phosphatase family of proteins containing an $\mathrm{Mg}(2+)$ dependent apurinic/apyrimidinic endonuclease Ape1-like domain. ${ }^{2}$ Various yeast-two hybrid $(\mathrm{Y} 2 \mathrm{H})$ screenings and functional studies have unveiled TTRAP ability to interact with TNF receptors (TNFR) family members and TNFR-associated factors (TRAFs), especially TRAF6. ${ }^{3}$ Furthermore, TTRAP was isolated as ETS-associated protein $~^{4}$ and as alk4- and smad3-binding protein. ${ }^{5}$ Interestingly, TTRAP sequence contains a SUMO-interacting motif (SIM) that is required for its high affinity binding to SUMO-2/3. ${ }^{6}$ TTRAP may thus has a role in the cytoplasm as a signaling molecule and in the nucleus as a DNA damage response protein and transcriptional regulator. In this context, its potential interaction with promyelocytic leukemia protein (PML), as previously determined in $\mathrm{Y} 2 \mathrm{H}$, suggests that TTRAP is a PML nuclear bodies (PML-NBs)-associated protein. ${ }^{7}$ It remains unclear whether its $5^{\prime}$-tyrosyl DNA phosphodiesterase activity may account for all TTRAP biological functions.

According to the cellular context, TTRAP may either protect cells from apoptosis or promote death. ${ }^{8-10}$ When human SH-SY5Y cells are stressed by low doses of proteasome inhibitors, TTRAP is neuroprotective. In these conditions, endogenous TTRAP relocalizes to the cytoplasm and forms aggresome-like inclusions.

In this paper we show that in response to high doses of proteasome inhibitors, TTRAP localizes to the nucleolus. This accumulation requires the association to PML-NBs and occurs in nucleolar cavities, a compartment devoid of markers of the 'ribosomal nucleolus'. Lack of TTRAP alters the levels of precursor ribosomal RNA (pre-rRNA) and processing intermediates, suggesting a new role of the PML-NBs-associated protein TTRAP in rRNA biogenesis. This function is dependent on TTRAP SIM motif, but is independent from its 5 'tyrosyl DNA phosphodiesterase activity. Altogether, these data suggest a novel function for TTRAP and nucleolar cavities in controlling rRNA biogenesis in response to proteasome inhibition.

\section{Results}

TTRAP accumulates in nucleolar cavities upon proteasome inhibition where it colocalizes with and binds to components of PML-NBs. Upon inhibition of proteasome-dependent protein degradation, proteins associated with PML-NBs accumulate in the nucleolus. In particular, PML itself, Sp100, SUMO-1 and p53 localize to subnucleolar regions in response to high doses of MG132. ${ }^{11-13}$ We previously showed that treatment of SH-SY5Y neuroblastoma cells with the low concentrations of proteasome inhibitors triggered TTRAP relocalization to the cytoplasm, to form juxtanuclear aggresome-like structures. ${ }^{8}$ We thus investigated the cellular localization of PML-NBs-associated protein TTRAP in the cells treated with increasing concentrations of drug. Localization to the nucleolus was assigned when TTRAP-positive granules

\footnotetext{
${ }^{1}$ Sector of Neurobiology, International School for Advanced Studies, Trieste, Italy; ${ }^{2}$ The Giovanni Armenise-Harvard Foundation Laboratory, Trieste, Italy; ${ }^{3}$ Laboratorio Nazionale Consorzio Interuniversitario Biotecnologie, Trieste, Italy; ${ }^{4}$ Department of Life Sciences (DSV), University of Trieste, Trieste, Italy and ${ }^{5}$ International School for Advanced Studies Unit, Italian Institute of Technology, Trieste, Italy

*Corresponding authors: S Zucchelli or S Gustincich, Sector of Neurobiology, International School for Advanced Studies, Via Bonomea 265, 34136 Trieste, Italy. Tel: + 390403787774 (SZ) or + 390403787705 (SG); Fax: + 390403787 702; E-mail: silvia.zucchelli@ sissa.it (SZ) or gustinci@ sissa.it (SG) Keywords: TTRAP; nucleolus; PML; proteasome inhibition; rRNA biogenesis

Abbreviations: TTRAP, TRAF and TNF receptor-associated protein; PML, promyelocytic leukemia; PML-NBs, PML nuclear bodies; Y2H, yeast-two hybrid; TRAF, TNF receptor-associated factor; rRNA, ribosomal RNA; pre-rRNA, precursor rRNA; DFC, dense fibrillar component; GC, granular component; NPM, nucleophosmin; NCL, nucleolin; UBF, upstream-binding factor; RAR $\alpha$, retinoic acid receptor alpha; SIM, SUMO-interacting motif

Received 01.2.11; revised 05.8.11; accepted 08.8.11; Edited by L Greene; published online 16.9.11
} 
were present in DAPI-negative subnuclear structures (Figure 1a). Nucleolar TTRAP was first detected after $8 \mathrm{~h}$ of MG132 treatment (Figure 1b) with the number of positive cells increasing over time. Nucleolar accumulation was strictly dependent on drug concentration as low amounts of MG132 $(0.25 \mu \mathrm{M})$ triggered TTRAP localization exclusively to cytoplasmic aggresome-like structures ${ }^{8}$ whereas nucleolar TTRAP was evident at higher doses (Figure 1b). In these conditions, TTRAP maintained its neuroprotective activity as measured with MTT assay (Figure 1c). In our experimental settings, the largest percentage of cells displaying nucleolar TTRAP was triggered by $5 \mu \mathrm{M}$ MG132 for $16 \mathrm{~h}$, with $\sim 60-70 \%$ of positive cells. As expected, TTRAP also accumulated into the cytoplasm as judged by biochemical fractionation (Figures $1 \mathrm{~d}$ and e). Nucleolar TTRAP was induced upon treatments with different proteasome inhibitors like lactacystin and epoxomicin, and was observed in all cell lines tested including HEK 293T, HeLa, U2OS, SAOS and IMR90 (data not shown).

To define the exact location of TTRAP-positive granules within the nucleolus, immunofluorescence experiments were carried out with specific markers for subnucleolar areas. First, we used nucleophosmin (NPM), as a marker of the granular component (GC), and nucleolin (NCL), for its localization in GC and in dense fibrillar component (DFC). As expected, NPM and NCL delineated the more external limits of the nucleolus (Figure 2a). TTRAP granules were encircled within the nucleolus, but did not colocalize with either NPM or NCL. When we compared TTRAP staining with fibrillarin and upstream-binding factor (UBF), labeling respectively the DFC and the fibrillar center, TTRAP did not colocalize with either of these proteins (Figure 2a). $z$-axis projection of triple immunofluorescence with TTRAP, NPM and UBF confirmed that TTRAP-positive granules are contained within nucleolar structures without any colocalization with canonical markers (Supplementary Figure S1). Taken together, these results suggest that TTRAP accumulates in a region that is devoid of markers for the 'ribosomal nucleolus', thus resembling nucleolar cavities. ${ }^{13}$

As the tumor suppressor p53 was recently shown to accumulate in nucleolar cavities upon treatment with MG132, ${ }^{13}$ TTRAP and p53 colocalization was studied in SH-SY5Y neuroblastoma cells. Upon proteasome inhibition, TTRAP granules colocalized with $\mathrm{p} 53$ and all cells containing nucleolar TTRAP also displayed nucleolar p53, thus restricting TTRAP accumulation to nucleolar cavities (Figure $2 b$ ). a

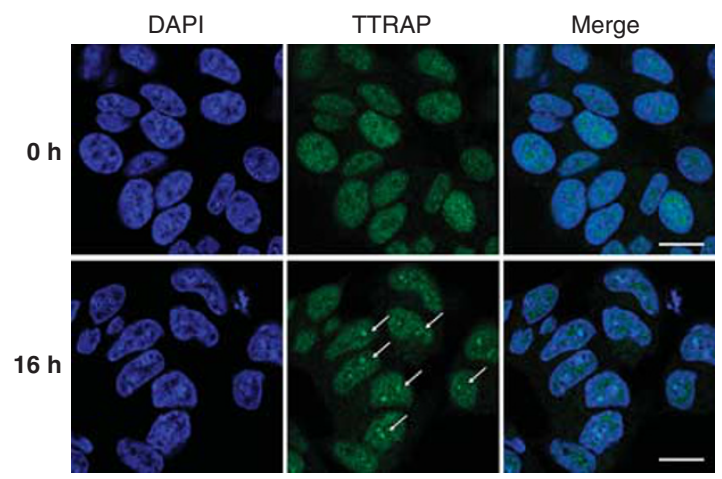

b

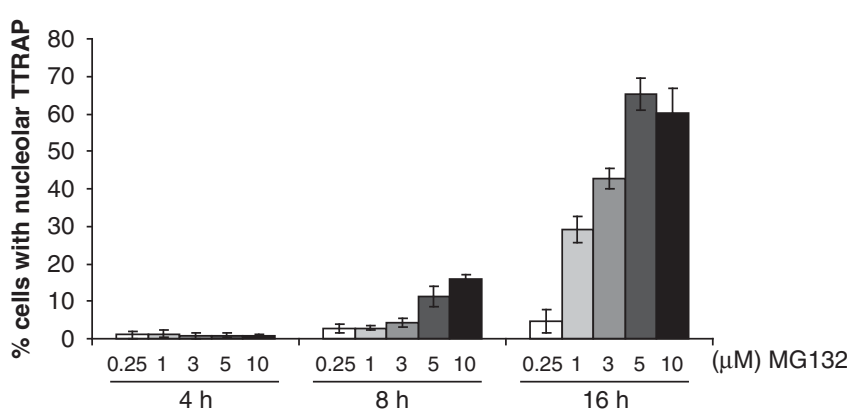

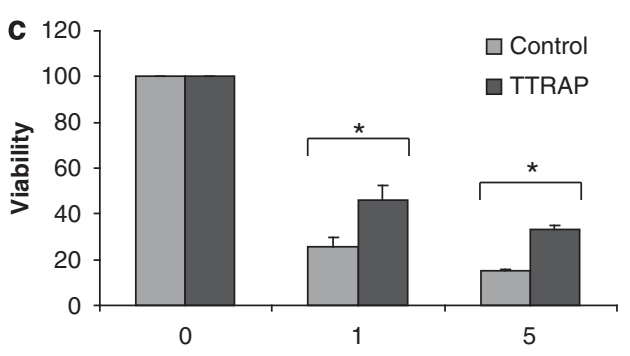

$(\mu \mathrm{M}) \mathrm{MG} 132$

d

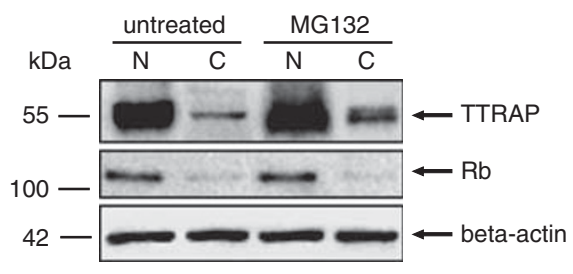

e

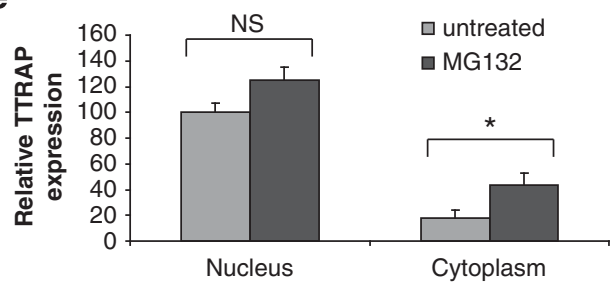

Figure 1 TTRAP accumulates in the nucleolus upon proteasome impairment. (a) Immunofluorescence of endogenous TTRAP in human neuroblastoma SH-SY5Y cells. Cells were treated with $5 \mu \mathrm{M}$ MG132 for $16 \mathrm{~h}$ or left untreated $(0 \mathrm{~h}$ ). TTRAP localization was analyzed by confocal immunofluorescence with anti-TTRAP (green). Nuclei were visualized with DAPI (blue). Arrows indicate TTRAP granules in DAPI-negative regions of the nucleus (nucleoli). Bars, $15 \mu \mathrm{m}$. (b) Bar chart showing the percentage of cells with nucleolar TTRAP. SH-SY5Y cells were treated with increasing doses of MG132 for the indicated time. Immunofluorescence was performed as in (a). At least 200 cells from two separate experiments were counted and scored for nucleolar TTRAP. (c) SH-SY5Y cells stably transfected with an empty vector (control) or with FLAG-TTRAP (TTRAP) were treated for $16 \mathrm{~h}$ with the indicated concentration of MG132. Cell viability was measured by MTT assay. Results are expressed as percentage of untreated cells. Data were collected from two independent TTRAP and control clones. Significance was calculated with $t$-test. ${ }^{*} P<0.05$. (d) Nuclear and cytoplasmic fractions from SH-SY5Y cells treated with $5 \mu \mathrm{M}$ MG132 or left untreated were biochemically separated. Protein lysates were analyzed by western blot with anti-TTRAP, anti-retinoblastoma (Rb) and anti- $\beta$-actin antibodies. (e) Quantification of relative TTRAP expression in nucleus and cytoplasm of SH-SY5Y cells upon proteasome inhibition was performed on high-resolution images from two independent experiments. NS, nonsignificant; ${ }^{*} P<0.05$ 


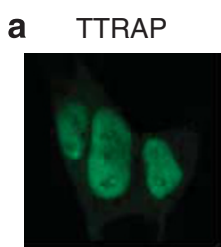

TTRAP

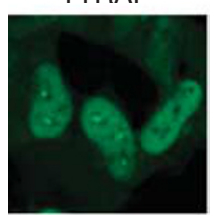

TTRAP

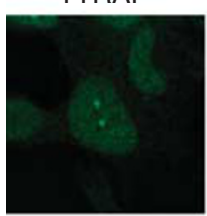

TTRAP

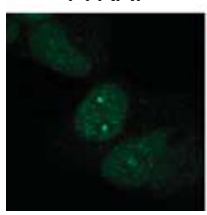

NPM

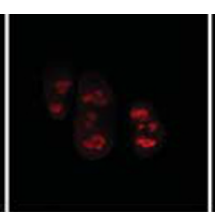

Nucleolin

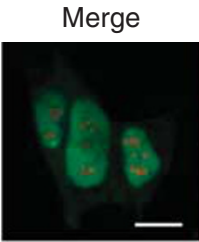

Merge

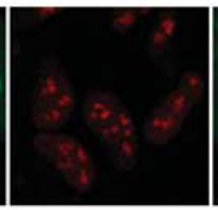

Fibrillarin

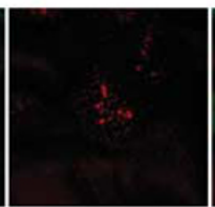

UBF
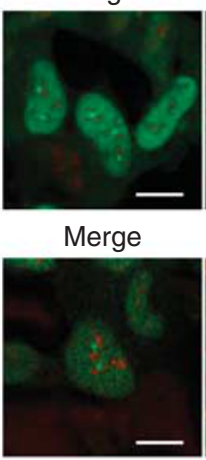

Merge

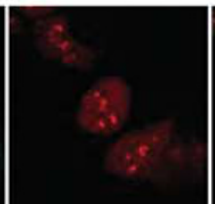

Enlargement

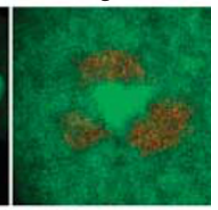

Enlargement

Enlargement
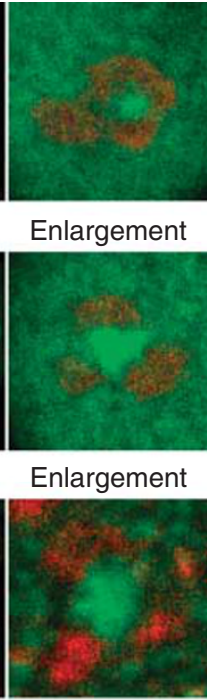

Enlargement
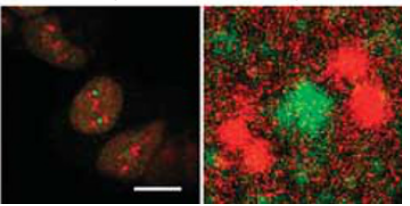

d

PML

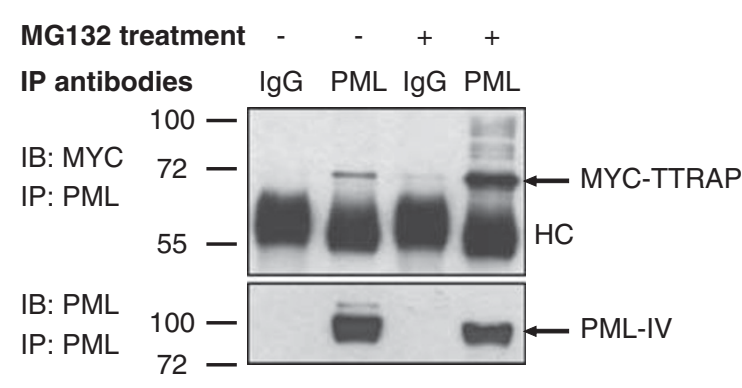

MG132 treatment - +

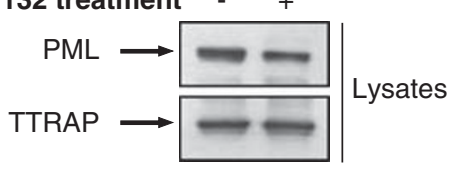

b
DAPI

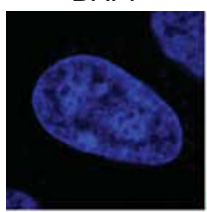

c NPM

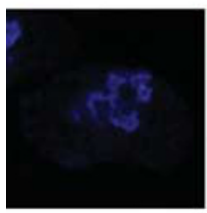

NPM

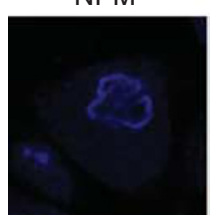

TTRAP

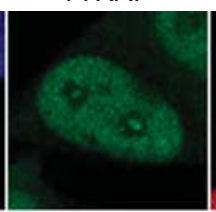

TTRAP

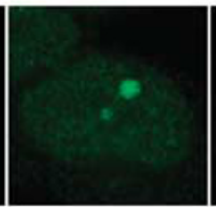

TTRAP

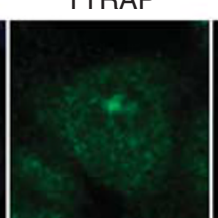

p53

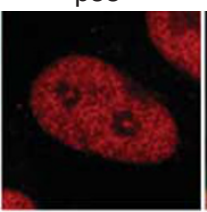

PML

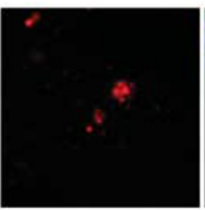

DAXX

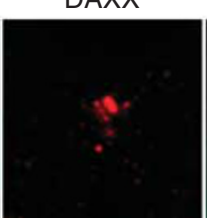

Merge

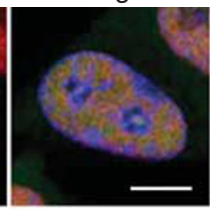

Merge

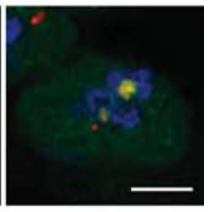

Merge

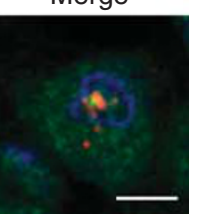

e
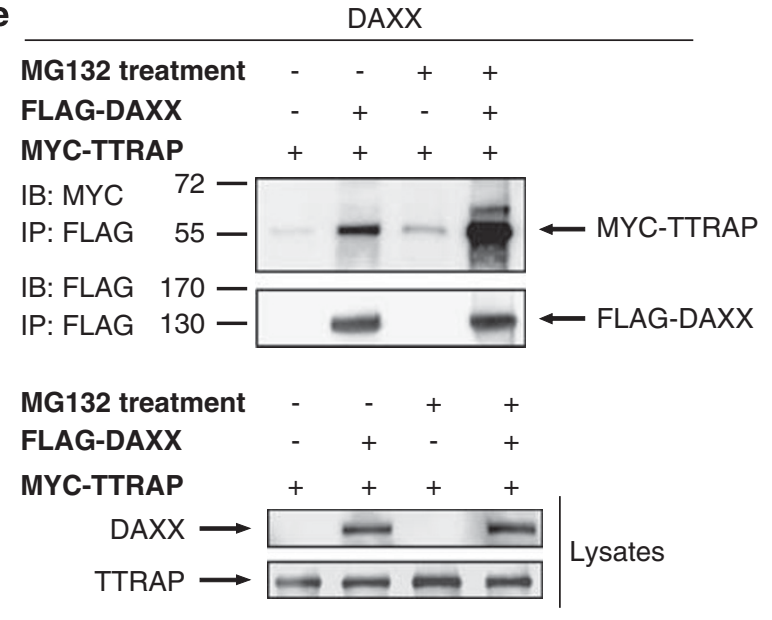

Figure 2 TTRAP associates with PML-NBs in nucleolar cavities and interacts with PML and PML-NBs component DAXX. (a) Nucleolar localization of TTRAP in MG132treated SH-SY5Y cells. SH-SY5Y cells were treated for $16 \mathrm{~h}$ with $5 \mu \mathrm{M}$ MG132. Double immunofluorescence was performed with anti-TTRAP (green) and nucleolar markers (red) NPM, NCL, Fibrillarin or UBF, as indicated. Colocalization is shown in merge. TTRAP location relative to nucleolar marker is highlighted in enlarged images. Bars, $10 \mu \mathrm{m}$. (b) TTRAP colocalizes with p53 in nucleolar cavities. SH-SY5Y cells were treated as in (a). Double immunofluorescence was performed with anti-TTRAP (green) and anti-p53 (red) antibodies. Colocalization between TTRAP and p53 is shown in merge. Bars, $5 \mu \mathrm{m}$. (c) TTRAP colocalizes with components of PML-NBs in the nucleolus. Cells as in (a). Triple immunofluorescence was performed with anti-NPM (blue), anti-TTRAP (green) and anti-PML or anti-DAXX (red) antibodies. Colocalization between the three proteins is shown in merge. Bars, $5 \mu \mathrm{m}$. (d) HEK-293T cells were transfected with MYC-TTRAP and PML IV. After transfection, cells were left untreated or incubated with $5 \mu \mathrm{M}$ MG132 for $16 \mathrm{~h}$, as indicated. Cell lysates were immunoprecipitated (IP) with anti-PML or control mouse lgG and bound proteins were revealed by immunoblot (IB) with anti-MYC and anti-PML antibodies. An aliquote of each lysate was tested for the expression of PML and TTRAP proteins, as indicated. Molecular weight markers are indicated on the left (KDa). HC, heavy chain. (e) HEK-293-T cells were transfected with FLAG-DAXX and MYC-TTRAP. Cells were treated as in (d). Cell lysates were immunoprecipitated with anti-FLAG agarose beads and bound proteins were revealed with anti-FLAG and anti-MYC antibodies

TTRAP was previously shown to interact with $\mathrm{PML}$ in $\mathrm{Y} 2 \mathrm{H}$ and to localize within PML-NBs in transfected cells. ${ }^{7}$ As proteins associated with PML-NBs accumulate in the nucleolus upon treatment with high concentration of proteasome inhibitors, ${ }^{11,12}$ we proved that TTRAP nucleolar granules were PML-NBs by immunofluorescence for TTRAP, NPM and pan-PML. Similar results were obtained with the PML-NBs-associated protein DAXX (Figure 2c). Interestingly, all TTRAP nucleolar granules colocalized with PML, but additional PML-positive and TTRAPnegative granules were formed in $10-15 \%$ of cells. Almost all of 
them were present in the nucleoli, but in a small percentage of cases (1-2\%) PML-positive and TTRAP-negative granules were in the nucleoplasm.

To confirm that the nucleolar TTRAP is a part of PML-NBs, we tested its physical interaction with PML-IV and PML-NBs component DAXX. Both proteins (Figures $2 d$ and e) were detectable in TTRAP-bound fractions but not in negative controls. The binding increased upon proteasome inhibition.

Double immunofluorescence with TTRAP and ubiquitin or 205 proteasome was also performed to assess whether nucleoli may be involved in an alternative degradation pathway of PML-NBs components. ${ }^{11}$ Ubiquitin was not accumulated in nucleoli upon MG132 treatment, and nucleolar TTRAP did not colocalize with proteasomes (Figure 3a). No colocalization was observed with components of other subnuclear structures like Cajal bodies (Figure $3 \mathrm{~b}$ ) or nuclear speckle (Figure 3c).

These results indicate that upon proteasome inhibition, TTRAP accumulates in nucleolar cavities where it colocalizes and physically interacts with PML-NBs-associated proteins.

TTRAP nucleolar localization requires binding to PML-NBs through SIM. We next investigated the role of PML expression and PML-NBs structure in TTRAP nucleolar localization. SH-SY5Y cells were transfected with vectors containing interference oligonucleotides against PML (siPML\#1) or scramble control and treated with MG132. Cells with reduced PML expression showed diffuse TTRAP nuclear staining but no nucleolar accumulation (Figure 4a). Nucleolar TTRAP was evident when a scramble control was used (Figure 4b). Similar results were obtained with an independent sequence to silence PML expression (siPML\#2), thus excluding off-target effects (Supplementary Figure S2).

The integrity of PML-NBs is disrupted in acute PML, in which PML is fused to retinoic acid receptor alpha (RAR $\alpha$ ). SH-SY5Y cells transfected with PML-RAR $\alpha$ showed the typical micro-speckled pattern of PML, while adjacent non-transfected cells maintained PML-NBs structure (Figure 4a). A marked impairment in TTRAP recruitment to the nucleolus was observed in cells with disorganized nuclear bodies, with no TTRAP accumulation in any PML-RAR $\alpha$ transfected cells (Figures $4 a$ and $b$ ). These data proves that PML has a pivotal role in the accumulation of TTRAP in the nucleolus in response to proteasome inhibition.

Interactions mediated by SUMO-binding motifs may represent a mechanism to modulate assembly of macromolecular structures such as PML-NBs. ${ }^{14}$ A SIM has been found in TTRAP sequence, and TTRAP can bind SUMO-2/3 with a higher affinity than SUMO-1. ${ }^{6}$ As PML itself can be SUMOylated by SUMO-3, ${ }^{15}$ we asked whether TTRAP SIM domain might have a role in recognition of PML and in PML-mediated TTRAP recruitment to nucleolar cavities. To address this issue, we generated a TTRAP SIM mutant unable to recognize SUMO-2/3 moieties. ${ }^{6} \mathrm{SH}-\mathrm{SY} 5 \mathrm{Y}$ cells with silenced TTRAP expression (siTTRAP cell) ${ }^{8}$ were reconstituted with TTRAP wt or SIM mutant constructs and treated with MG132. As expected, cells transfected with wt TTRAP displayed the typical nucleolar accumulation (Figure 4c). The expression of SIM mutant showed an aberrant cytoplasmic localization and a reduced, but maintained, nuclear staining. Importantly, mutagenesis of TTRAP SIM domain completely abrogated the accumulation of TTRAP in the nucleolus. The absence of TTRAP nucleolar staining was not due to reduced protein levels as determined by western blot analysis (data not shown).

We then asked whether the SIM domain was required for TTRAP nucleolar localization via its physical interaction with PML. While TTRAP wt efficiently bound PML, SIM mutant did not (Figure 4d), thus, proving that TTRAP SIM motif is essential for TTRAP binding to PML and localization into nucleolar cavities during proteotoxic stress. Interestingly, we observed no changes in PML or DAXX nucleolar localization in siTTRAP SH-SY5Y cells (Supplementary Figure S3), and overexpression of TTRAP SIM mutant had no effect on such phenotype (data not shown).

\section{A functioning nucleolus is required for TTRAP} localization in nucleolar cavities. We then asked whether a functioning nucleolus is required for TTRAP nucleolar localization. To this purpose, we used low concentrations of actinomycin $D(0.08 \mu \mathrm{g} / \mathrm{ml})$ to specifically block RNA polymerase I and consequently rRNA synthesis. ${ }^{16} \mathrm{SH}-\mathrm{SY} 5 \mathrm{Y}$ treated with MG132 alone displayed nucleolar TTRAP in $60-70 \%$ of cells, as expected. Inhibition of rRNA transcription caused a statistically significant decrease in the number of cells with nucleolar TTRAP $(10-15 \%$ reduction; Figures $5 \mathrm{a}$ and $\mathrm{b})$. The effect of actinomycin $D$ was specific, as a dose-dependent reduction of TTRAP nucleolar granules was observed with increasing concentration of the drug. No changes in TTRAP protein levels were observed at any treatment (data not shown).

By adding higher concentrations of actinomycin $D$, nucleolar structures were dispersed (Figure 5a) and TTRAP nucleolar granules almost disappeared (Figure 5b). Exclusion of NPM from the nucleolus was observed when cells were treated with only high dose of actinomycin $D$, as expected (Figure 5a). Treatment with $\alpha$-amanitin $(50 \mu \mathrm{g} / \mathrm{ml})$, an inhibitor of RNA polymerases II- and III-mediated transcription, did not affect TTRAP nucleolar relocalization (data not shown). Therefore, intact nucleoli with active rRNA transcription are required for TTRAP nucleolar localization upon proteasome inhibition.

TTRAP regulates levels of rRNA biogenesis in conditions of proteasome inhibition. The main function of the nucleolus is ribosome biogenesis, for which fundamental steps are transcription and processing of rRNA molecules. To study the role of nucleolar TTRAP in rRNA biogenesis, we first analyzed the levels of pre-rRNAs by quantitative real-time PCR (qPCR), with oligonucleotides targeting $5^{\prime} \mathrm{ETS}(\mathrm{A0}) .{ }^{17} \mathrm{~A}$ similar qPCR strategy was then carried out to monitor the levels of intermediates by designing oligonucleotides that target regions of pre-rRNA eliminated during the processing steps. In particular, we selected cleavage sites 1 and 4 that are common to multiple alternative processing pathways (Figure 6a). rRNA biogenesis was measured by qPCR with $\mathrm{A} 0,1$ and 4 primers in siTTRAP or control cells after treatment with MG132. To exclude off-target effects, all experiments were repeated in two independent cell lines (\#1 and \#2) with 


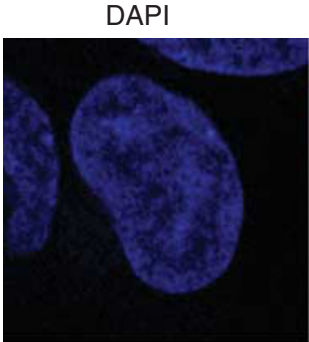

DAPI

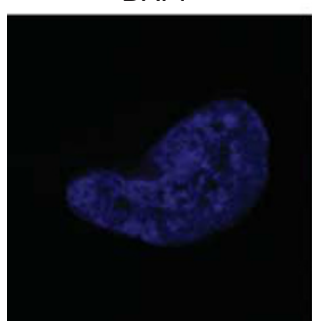

Ubiquiti
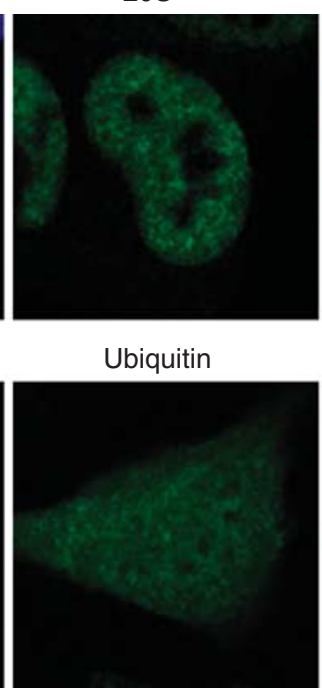

TTRAP

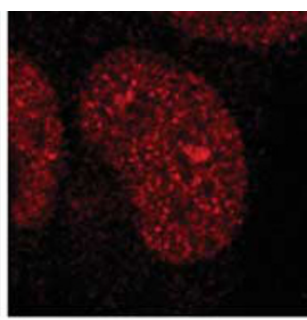

TTRAP
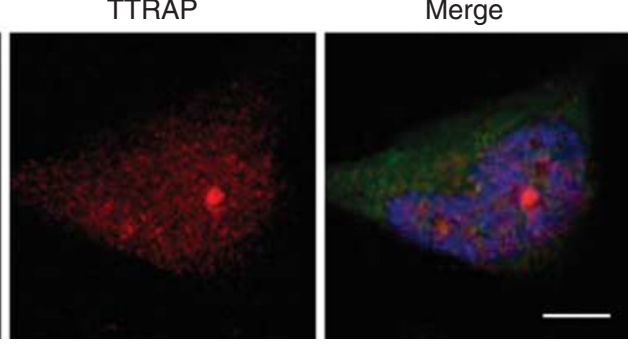

b

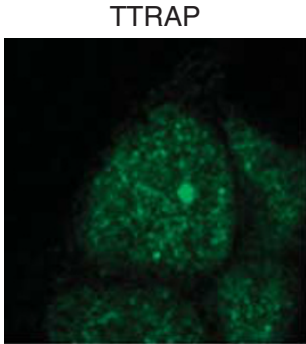

TTRAP

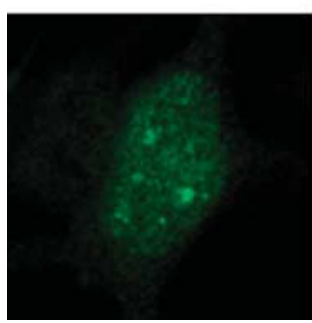

C

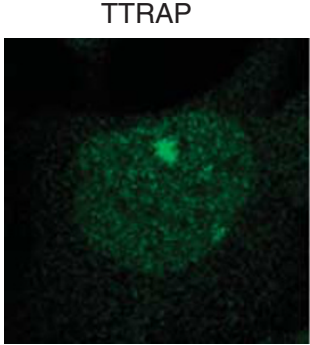

Coilin

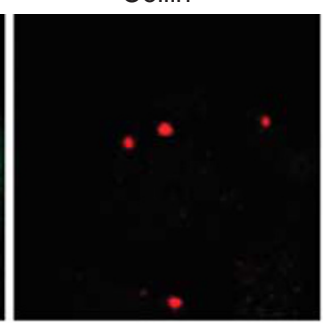

FLASH

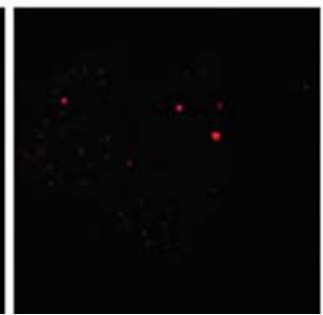

SC35

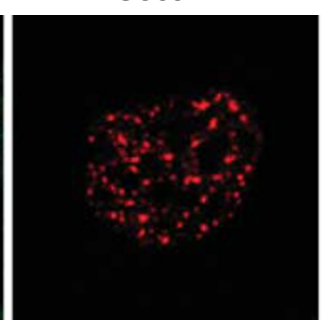

Merge

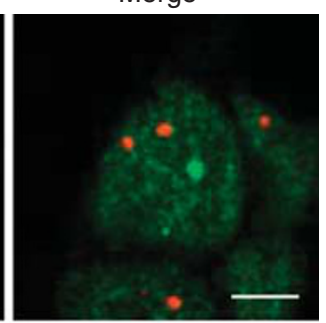

Merge

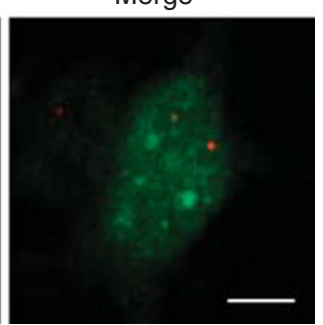

Merge

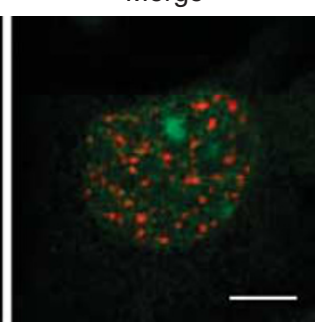

Figure 3 TTRAP-positive nucleolar cavities do not accumulate proteins of ubiquitin proteasome system, Cajal bodies or splicing speckles. (a) Human SH-SY5Y cells were treated with $5 \mu \mathrm{M}$ MG132 for $16 \mathrm{~h}$. Double immunofluorescence was performed with anti-TTRAP (green) and anti-20S or anti-Ubiquitin (red) antibodies. (b) Double immunostaining of endogenous TTRAP (green) with components of Cajal bodies (FLASH and Coilin, as indicated) on SH-SY5Y cells treated as in (a). (c) SH-SY5Y cells were treated as in (a). Immunofluorescence with anti-TTRAP (green) and anti-SC35 (red), as marker of splicing speckles. Bars, $5 \mu \mathrm{m}$

silencing sequence targeting different regions of TTRAP mRNA. $^{8}$ In untreated conditions, $\mathrm{A} 0,1$ and 4 probes detected no changes dependent on TTRAP silencing (Figure 6b). Upon proteasome inhibition, the levels of prerRNA containing AO sites were significantly increased.
This induction was strongly reduced in siTTRAP cells. Furthermore, the amount of rRNA processing intermediates, measured by oligonucleotides targeting 1 and 4 cleavage sites, dramatically increased in siTTRAP cells compared with controls. 
a
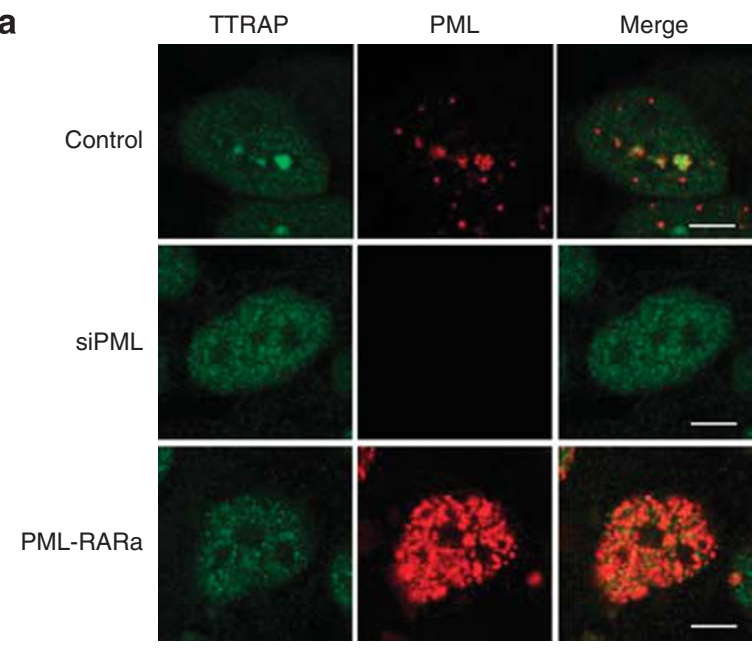

c
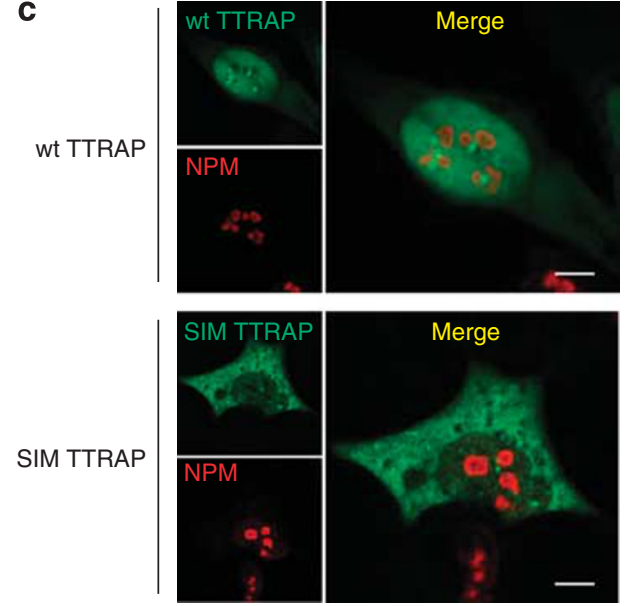

b

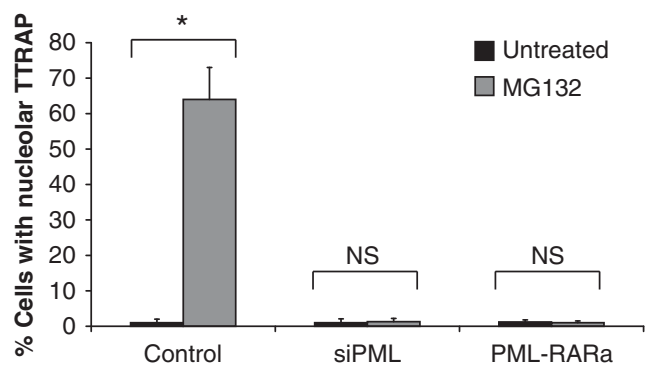

d
Untreated

$\square$ MG132

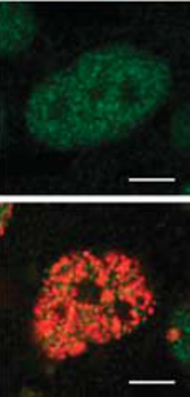

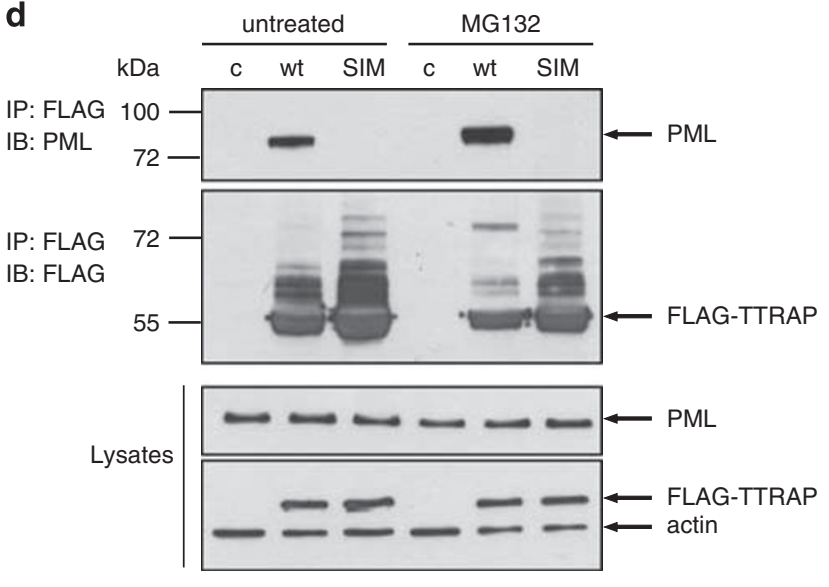

Figure 4 PML-NBs are required for TTRAP nucleolar localization. (a) SH-SY5Y cells were transfected with scramble control, siPML\#1 or PML-RARalpha (PML-RAR $\alpha$ ) constructs as indicated, and treated for $16 \mathrm{~h}$ with $5 \mu \mathrm{M}$ MG132. Immunofluorescence was performed using anti-TTRAP (green) and anti-PML (red) antibodies. Images are representatives of three independent experiments. Bars, $5 \mu \mathrm{m}$. (b) Quantification of TTRAP nucleolar staining. A total of 100 cells per condition were counted from three independent experiments. The data indicate mean \pm S.D. Significance between untreated and treated group was calculated with $t$-test $\left({ }^{*} P<0.01\right)$. NS, nonsignificant. (c) TTRAP SIM mutant does not accumulate in the nucleolus. siTTRAP SH-SY5Y cells were transfected with FLAG-TTRAP wt or SIM mutant and treated for $16 \mathrm{~h}$ with $5 \mu \mathrm{M}$ MG132. Double immunofluorescence was performed with anti-FLAG (green) and anti-NPM (red) antibodies. Images are representatives of at least three independent experiments performed on two siTTRAP clones. Bars, $5 \mu \mathrm{m}$. (d) TTRAP SIM domain is required for binding to PML. HEK-293T cells were transfected with PML and FLAGTTRAP wt or SIM mutant or empty vector as control and treated with MG132 $5 \mu \mathrm{M}$ for $16 \mathrm{~h}$ or left untreated. Overexpressed TTRAP was immunoprecipitated with anti-FLAG agarose beads. Co-immunoprecipitated PML was revealed with anti-PML antibody. Efficacy of immunoprecipitation was controlled in the same membrane with anti-FLAG antibody. Expression of proteins in cell lysates was verified with anti-FLAG and anti-PML. Loading was checked with anti- $\beta$-actin

To further investigate the role of nucleolar TTRAP on rRNA processing, we measured nascent rRNA in siTTRAP and scramble control cells by pulse-chase labeling with ${ }^{32} \mathrm{P}$-orthophosphate. Untreated cells were used as negative controls. Metabolically labeled rRNA species were visualized by autoradiography (Figure 6c, upper panel). Loading was verified with ethidium bromide (Figure 6c, lower panel). We followed transcription and processing of nascent rRNA with $1 \mathrm{~h}$ and $3 \mathrm{~h}$ chase. As expected, at $1 \mathrm{~h}$ we could observe initial accumulation of precursors (47S/45S) and processing intermediates (32S). At $3 \mathrm{~h}$, mature forms appeared with the mature $28 \mathrm{~S}$ rRNA typically more abundant than the $18 \mathrm{~S}$. No differences in rRNA processing were detected in untreated cells between scramble and siTTRAP cells. Upon proteasome inhibition, silencing TTRAP expression caused a reduction in pre-rRNA precursors (47S/45S) and an accumulation of the $32 S$ processing intermediate. These data suggest that TTRAP is involved both in the transcription of precursor $47 S / 45 S$ and in the conversion of the $32 S$ intermediate to the mature $28 \mathrm{~S}$ rRNA form.

In summary, silencing TTRAP expression causes alterations in rRNA biogenesis during proteasome impairment, with a decrease of pre-rRNA and an accumulation of processing intermediates.

TTRAP SIM motif but not 5'-tyrosyl DNA phosphodiesterase activity is essential for its role in ribosome biogenesis. We asked whether TTRAP accumulation in 
a

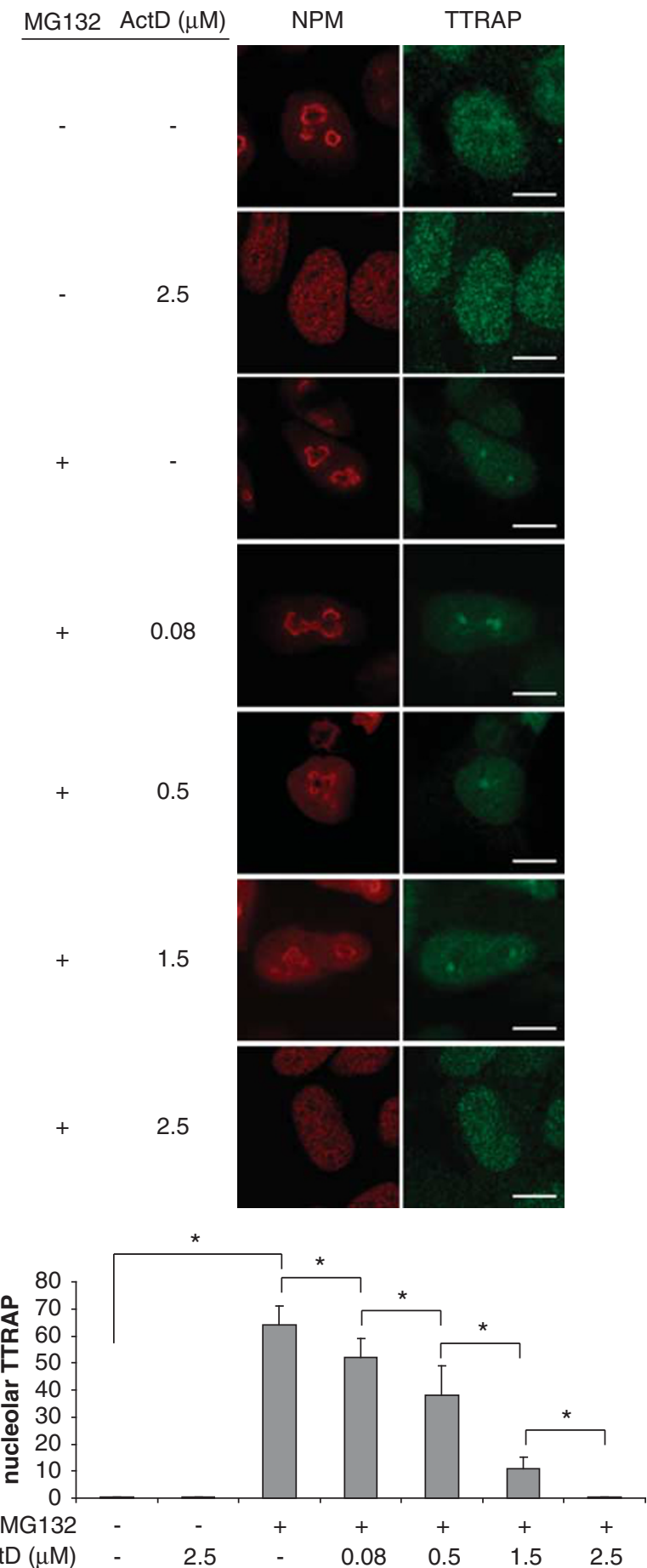

Figure 5 TTRAP accumulation in the nucleolus requires active rRNA transcription. (a) SH-SY5Y cells were treated with MG132 $5 \mu \mathrm{M}$ for $16 \mathrm{~h}$, then the medium was replaced and the cells were treated with increasing concentrations of actinomycin $D(A c t D)$, as indicated. Control cells were either left untreated or treated with ActD alone. Indirect double immunofluorescence was performed with antibodies against TTRAP (green) and NPM (red). Bars, $5 \mu \mathrm{m}$. (b) Quantification of cells containing TTRAP-positive nucleolar granules. The percentage of cells with nucleolar TTRAP was estimated by counting at least 100 cells for each experiment, in three separate experiments. Significance was calculated with $t$-test $\left({ }^{*} P<0.05\right)$
PML-NBs in the nucleolus is required for its ability to regulate rRNA processing. Therefore, we reconstituted siTTRAP cells with wt or SIM mutant and followed rRNA biogenesis in conditions of proteotoxic stress. Expression of wt TTRAP was able to partially rescue the alteration in rRNA biogenesis due to TTRAP silencing (Figure 7a) to levels compatible with low transfection efficiency in SH-SY5Y cells. These results further confirm that alterations in rRNA molecules are not due to off-target effects. TTRAP SIM mutant was not able to revert the phenotype, indicating that the SIM domain and nucleolar localization are required for TTRAP to function (Figure 7a). This effect was not due to different levels of expressed proteins, as the amounts of wt and SIM TTRAP were comparable. As expected, no detectable changes in steady-state levels of pre-rRNA and processing intermediates were observed after overexpression of wt or SIM mutant TTRAP in the absence of MG132 treatment (Supplementary Figure S4).

We then asked whether $5^{\prime}$-tyrosyl DNA phosphodiesterase activity is required for TTRAP to regulate rRNA biogenesis through a DNA repair-associated function. siTTRAP cells were then reconstituted with constructs for phosphodiesterase mutants E152A and D262A. ${ }^{1}$ No detectable changes in pre-rRNA or processing intermediates were observed in the absence of MG132 treatment with all transfected constructs (Supplementary Figure S4). Notably, phosphodiesterase inactive TTRAP (both E152A and D262A mutants) was able to rescue the alterations in rRNA biogenesis and this effect was comparable to that of wt protein (Figure $7 b$ ), thus suggesting that the enzymatic activity of TTRAP is dispensable for its control on rRNA maturation. Accordingly, TTRAP phosphodiesterase mutants were able to localize in the nucleolus as wt TTRAP (Figure 7c). Consistent with these results, we found that TTRAP-containing granules in nucleolar cavities are not DNA repair foci (Figure 8a). Furthermore, etoposide treatment did not trigger TTRAP nucleolar relocalization. However, when etoposide was followed by proteasome block, TTRAP-containing DNA damage foci were formed proving that TTRAP can be involved in DNA damage repair also in neuroblastoma cells (Figure $8 b$ ).

Altogether these results indicate that TTRAP localization in the nucleolus via its SIM motif is essential to preserve normal rRNA biogenesis after proteasome block and this activity does not involve its DNA repair function.

\section{Discussion}

In this paper we demonstrate that PML-NB-associated TTRAP is found in the nucleolus in human neuroblastoma cells treated with proteasome inhibitors. In the nucleolus TTRAP accumulates in the recently defined nucleolar cavities, where it colocalizes with p53 and with components of PML-NBs. PML itself and TTRAP-SIM motif are required for sequestration of TTRAP in the cavities. In the nucleolus TTRAP contributes to regulate rRNA biogenesis and its $5^{\prime}$-phosphodiesterase activity is dispensable for its nucleolar function.

rRNA biogenesis is a highly complex process that requires the transcription of rDNA genes by polymerase I, nucleolytic cleavages and chemical modification of pre-rRNAs and 


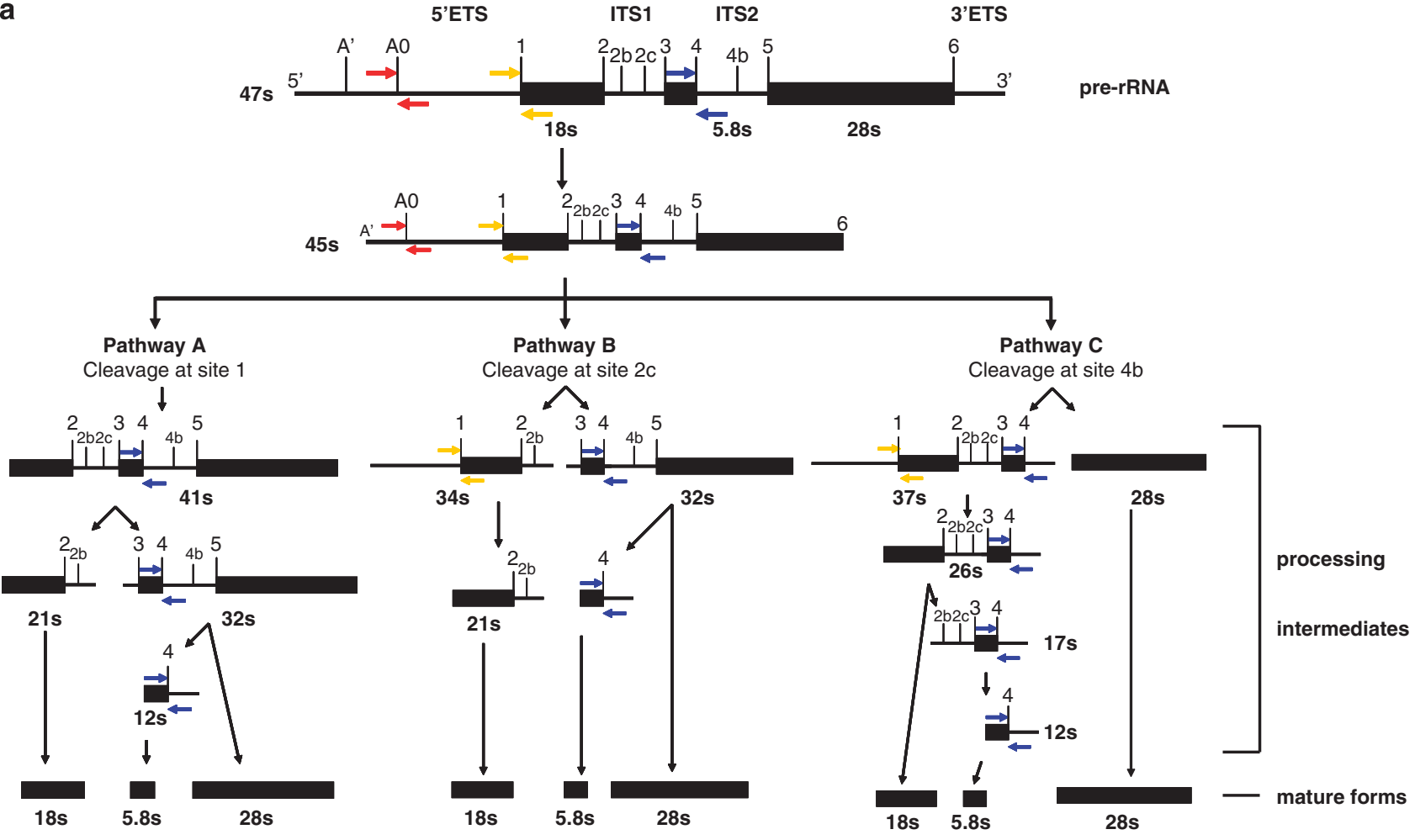

b

\begin{tabular}{|c|c|c|c|c|c|c|c|}
\hline C & & & & & UG & & \\
\hline RAP & - & + & + & - & & + & + \\
\hline Chase (h) & 1 & 1 & 3 & 1 & 3 & 1 & 3 \\
\hline
\end{tabular}
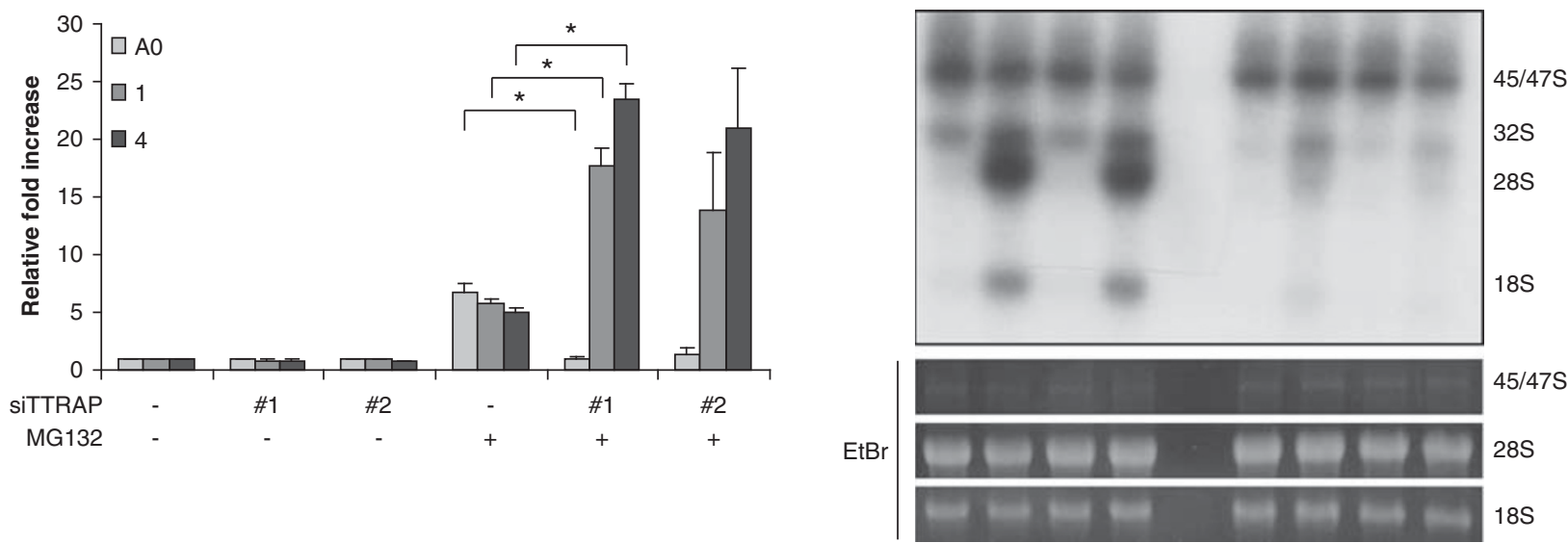

Figure 6 TTRAP regulates rRNA processing upon proteasome inhibition. (a) Schematic diagram showing pre-rRNA structure and processing steps, modified from Morello et al. ${ }^{40} \mathrm{~A} 0,1$ and 4 are sites monitored by qPCR. ETS, external transcribed spacer; ITS, internal transcribed spacer. (b) siTTRAP (\#1 and \#2 clones) or control cells were treated with $5 \mu \mathrm{M}$ MG132 for $16 \mathrm{~h}$ or left untreated. Total RNA was extracted, and rRNA biogenesis was analyzed by qPCR relative to $\beta$-actin and GAPDH housekeeping genes. The value given for the amount of PCR product present in control cells untreated was set as 1 . Standard deviations are calculated from two independent experiments. ${ }^{*} P<0.05$. (c) siTTRAP and control cells were treated with $5 \mu \mathrm{M}$ MG132 for $16 \mathrm{~h}$ or left untreated. After treatment, cells were pulse labeled with ${ }^{32} \mathrm{P}$-orthophosphate for $1 \mathrm{~h}$ and chased with cold medium for $1 \mathrm{~h}$ or $3 \mathrm{~h}$. RNA was extracted and separated on denaturing agarose gel. Ethidium bromide staining (lower panel) and autoradiography after gel drying (upper panel) are shown

ultimately leads to the assembly of mature ribosomes. ${ }^{18}$ It is subjected to stringent quality control mechanisms ${ }^{19,20}$ and stressful events elicit homeostatic responses that involve the majority of its steps. ${ }^{21,22}$

Several human genetic diseases, collectively defined as ribosomopathies, are caused by mutations in genes that affect rRNA biogenesis and ribosome assembly. Congenital muta- tions in RPS19 and other genes encoding ribosomal proteins have been linked to Diamond-Blackfan anemia, a disorder characterized by a reduction of erythroid precursors. ${ }^{23}$ Mutations in other genes required for normal ribosome biogenesis have been implicated in other rare congenital syndromes such as Treacher Collins syndrome, dyskeratosis congenita, cartilage-hair hypoplasia and 


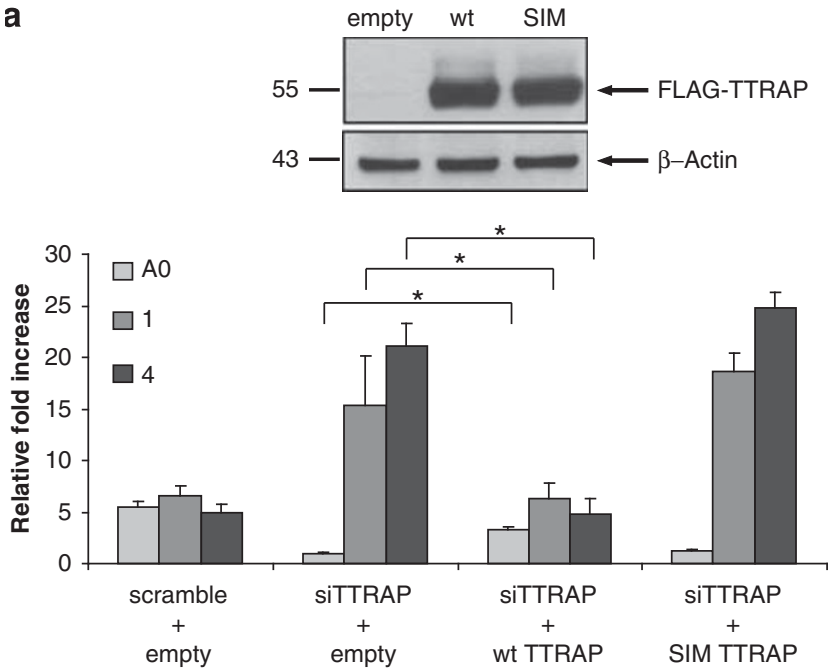

b

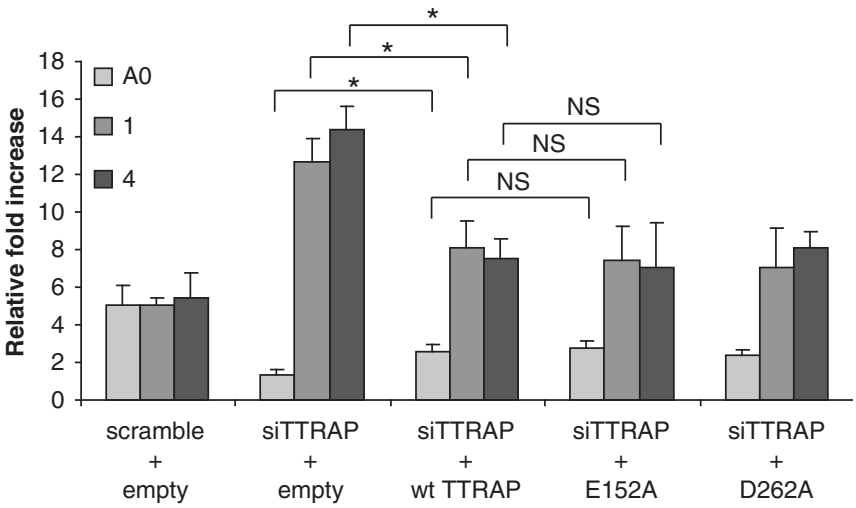

C

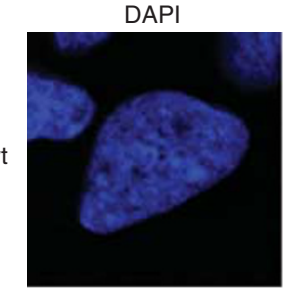

TTRAP
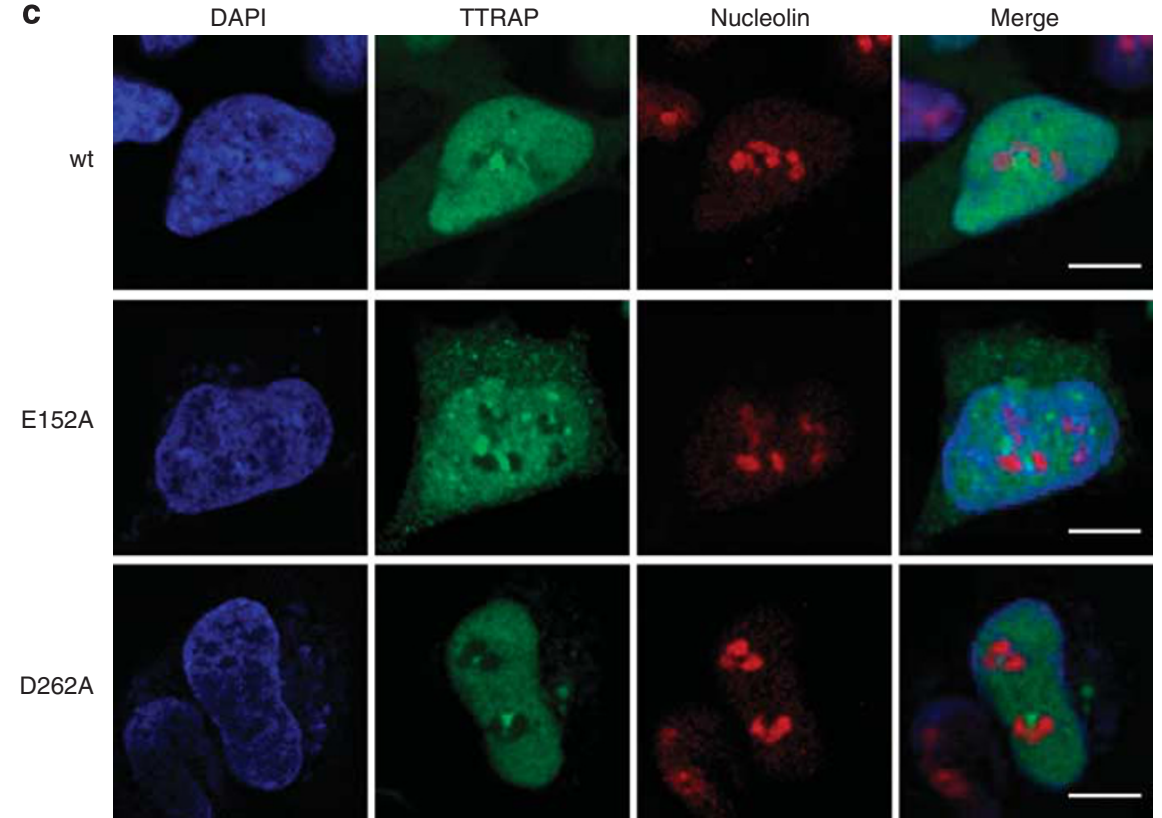

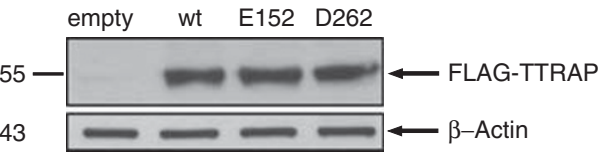

E152A

Figure 7 TTRAP SIM motif but not $5^{\prime}$-tyrosyl DNA phosphodiesterase activity is essential for its role in rRNA biogenesis. (a) siTTRAP-stable cells were transfected with FLAG-TTRAP wt or SIM mutant or empty vector as control and treated with $5 \mu \mathrm{M}$ MG132 for $16 \mathrm{~h}$. rRNA biogenesis was followed by qPCR relative to $\beta$-actin and GAPDH housekeeping genes. Standard deviation was calculated from three independent experiments. Significance between siTTRAP cells transfected with wt TTRAP or with empty vector was calculated with $t$-test $\left({ }^{*} P<0.05\right)$. NS, nonsignificant. In the same experiment, cell lysates were prepared and analyzed by immunoblot for the presence of ectopic TTRAP protein. $\beta$-Actin was used as loading control. (b) siTTRAP-stable cells were transfected with TTRAP wt or phosphodiesterase mutants E152A or D262A or empty vector as control and processed as in (a). (c) TTRAP E152A and D262A mutants do not accumulate in the nucleolus. SiTTRAP-stable SH-SY5Y cells were transfected and treated as in (b). Double immunofluorescence was performed with anti-FLAG and anti-NCL antibodies. Images are representatives of two independent experiments Bars, $7 \mu \mathrm{m}$

Schwachman-Diamond syndrome. ${ }^{24}$ Acquired abnormalities in ribosome function have also been implicated more broadly in human malignancies. ${ }^{25}$

Although it is accepted that proteotoxic stress triggers substantial alterations in rRNA biogenesis, its specific effects are variable depending on drug concentrations, cell lines and kinetics of treatments. ${ }^{21,26,27}$ Transcription and processing of pre-rRNA require a plethora of proteins and enzymatic activities to generate mature molecules. By qPCR and in vivo pulse labeling we found TTRAP regulates rRNA biogenesis exclusively under proteasome impairment. This is in agreement with the observation that knocking down genes essential for rRNA synthesis in physiological conditions impairs cell proliferation, ${ }^{24}$ while silencing TTRAP expression in the absence of proteasome block has no effects on growth of neuroblastoma cells (data not shown).

Primers targeting A0, 1 and 4 cleavage sites were chosen to monitor collectively rRNA biogenesis, including transcription by RNA polymerase I, rRNA maturation and degradation of cleaved fragments. In Diamond-Blackfan anemia and other 
DAPI

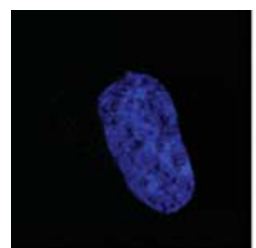

DAPI

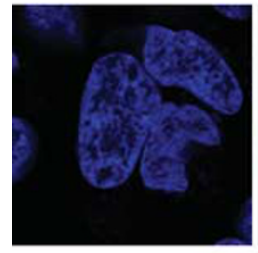

DAPI

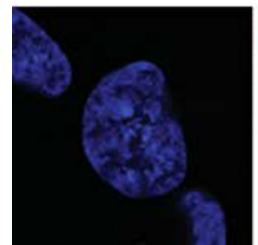

b
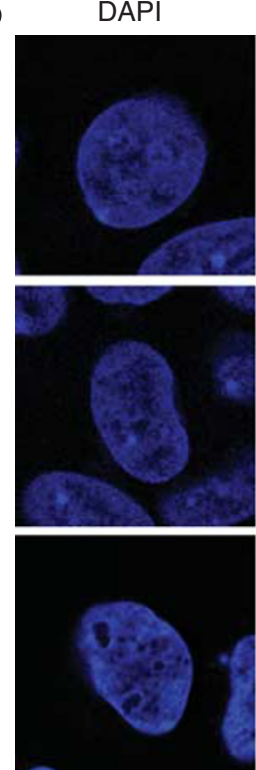

TTRAP

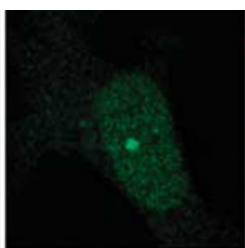

TTRAP

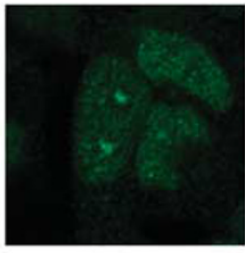

TTRAP
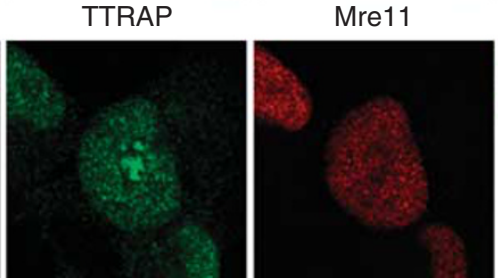

NPM
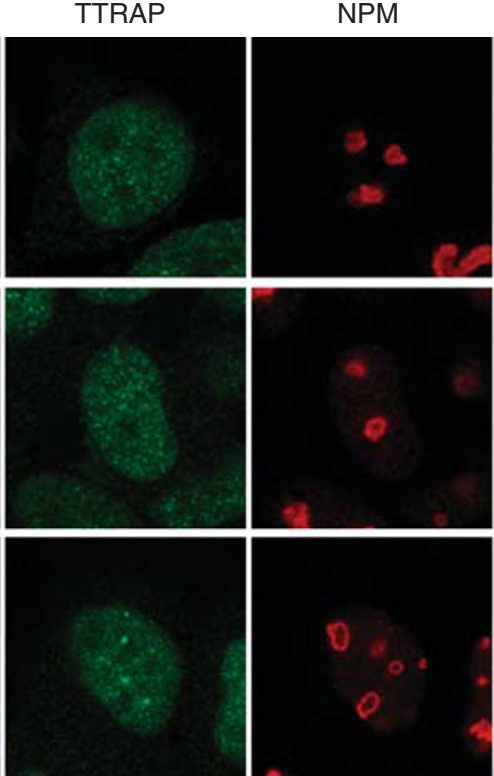

ATM

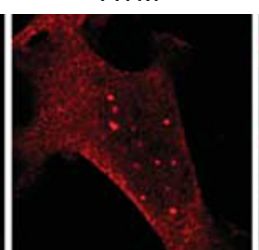

gamma-H2AX

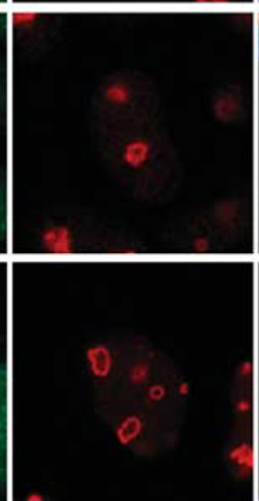

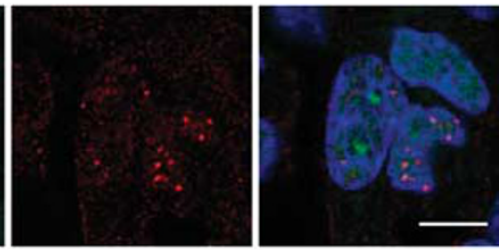

Merge

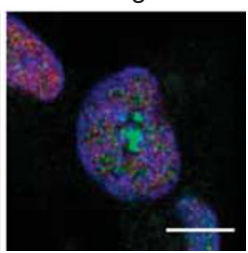

Merge

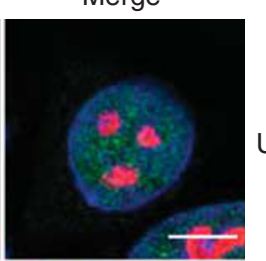

Untreated

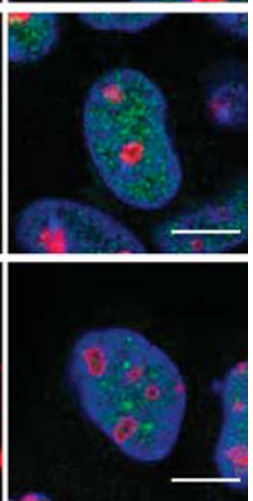

Etoposide

Etoposide + MG132

Figure 8 TTRAP nucleolar granules do not colocalize with DNA damage foci. (a) Human SH-SY5Y cells were treated with $5 \mu \mathrm{M}$ MG132 for $16 \mathrm{~h}$. Double immunofluorescence was performed with anti-TTRAP (green) and anti-ATM or anti- $\gamma-\mathrm{H} 2 \mathrm{AX}$ or anti-Mre11 (red) antibodies. (b) Immunofluorescence of endogenous TTRAP in human neuroblastoma SH-SY5Y cells. Cells were treated with etoposide $100 \mu \mathrm{M} 6 \mathrm{~h}$ or with etoposide $100 \mu \mathrm{M}$ plus MG132 $5 \mu \mathrm{M}$ for $6 \mathrm{~h}$ or left untreated. TTRAP localization was analyzed by confocal immunofluorescence with anti-TTRAP (green) and anti-NPM (red). Nuclei were visualized with DAPI (blue). Bars, $7 \mu \mathrm{m}$

ribosomopathies, deletion of a ribosomal protein that is involved exclusively in rRNA processing leads to the increase of a specific intermediate form without alterations in pre-rRNA levels. ${ }^{28}$ On the other hand, absence of factors essential for rRNA transcription or treatment with drugs that inhibit RNA polymerase I has a negative impact on the levels of both prerRNA and processing intermediates. ${ }^{26}$ One can speculate that TTRAP might function at multiple steps, as downregulation of TTRAP leads to a decrease of pre-rRNA and a concomitant increase of processing species. Accumulation of processing intermediates might be due to impaired cleavage or to inhibition of degradation of cleaved fragments. As TTRAP 5'-tyrosyl DNA phosphodiesterase activity seems to be dispensable for TTRAP nucleolar function, we favor the hypothesis that TTRAP protein-protein interaction network, not its enzymatic activity, might be important. It has been recently found that the SUMO system controls partitioning between the nucleus and the nucleolus of a novel multiprotein 
complex that regulates ribosome biogenesis. ${ }^{29}$ These data support our model in which SUMO binding controls TTRAP nucleolar localization and activity as a part of cellular responses to stressors. Importantly, proteasome impairment also modifies the architecture of the nucleolus with the appearance of more complex shapes and less delineated GC. In the majority of cases, TTRAP and PML-NBs do not colocalize with any canonical nucleolar markers. As all these antigens label molecules and apparatus involved in rDNA transcription and ribosome biogenesis, we have identified this 'non-ribosomal area' as nucleolar cavities. ${ }^{13,30}$ They correspond to nucleoplasmic spaces within the nucleolus that may increase dramatically in number and size when mammalian cell lines undergo proteasome block. Electron microscopic examination revealed that they contain several distinct $\sim 40 \mathrm{~nm}$ particles of relatively high contrast, resembling perichromatin granules. ${ }^{13}$ Although their biological function remains largely unknown, in this paper we provide the first evidence for a role of nucleolar cavities in ribosome biogenesis. Furthermore, despite that all TTRAP-positive nucleolar structures contain PML, some PML-NBs are negative for TTRAP, suggesting the existence of different nucleolar cavities-associated PML-NBs.

Previous studies have shown that rRNA precursors accumulate in strains defective for exosome components. ${ }^{19}$ The exosome is the nucleolus-associated machinery involved in many aspects of RNA quality control and surveillance ${ }^{31}$ as well as in rRNA processing. ${ }^{32}$ In an $\mathrm{Y} 2 \mathrm{H}$ screen, TTRAP was found to bind to a regulatory subunit of yeast analogue of the human exosome (BIND database). It will be interesting to study whether nucleolar cavities are sites for exosome activity and TTRAP might contribute to regulate exosome function.

Stress-dependent regulation of rRNA biogenesis occurs mainly by influencing the transcriptional rate of active rDNA loci, but recent findings also point toward epigenetic regulation of rDNA repeats for the formation of transcriptionally active or silent loci. ${ }^{33,34}$ Our preliminary data indicate that TTRAP associates to histone deacetylase HDAC1 and HDAC3 and to co-repressor complex Sin3A, and these bindings are enhanced upon proteasome inhibition. All these proteins have been found in the nucleolar proteome. ${ }^{35}$ It will be interesting to assess the role of TTRAP in chromatin remodeling at rDNA loci in physiological and pathological conditions. Alternatively, TTRAP-mediated downregulation of pre-rRNA levels may be secondary to its role in rRNA processing, as already postulated for other non-ribosomal proteins involved in rRNA biogenesis. $^{36}$

In summary, these data provide the first evidence for the involvement of nucleolar cavities and TTRAP in ribosome biogenesis and support the need for a better understanding of their roles in cellular responses to stress in neurodegeneration and oncogenesis.

\footnotetext{
Materials and Methods

Cell culture, transfections and viability assay. Human neuroblastoma SH-SY5Y cells (ATCC) were maintained in culture as suggested by the vendor. SH-SY5Y cells stably transfected with pCDNA3-FLAG-TTRAP or empty vector control and with pSuperior-siTTRAP (\#1 and \#2 clones) or pSuperior-Scramble were previously described. ${ }^{8} \mathrm{All}$ stably transfected SH-SY5Y cells were kept in culture with neomycin selection. HEK-293T were grown in DMEM supplemented with 10\% FCS
}

and antibiotics. Transfections of HEK-293T cells were performed with standard calcium phosphate method. SH-SY5Y cells were transfected with Lipofectamine 2000 plus Optimem (Invitrogen, Carlsbad, CA, USA), according to manufacturer's instructions. For treatments, the following reagents were used: for proteasome inhibition MG132 (z-Leu-Leu-al), epoxomycin and lactacystin; for transcription inhibition actinomycin $D$ and $\alpha$-amanitin; for DNA damage etoposide. Reagents for treatments were all purchased from Sigma Aldrich (St. Louis, MO, USA).

Cell viability was measured using MTT Formazan (Sigma Aldrich) assays, following manufacturer's instructions and reading absorbance at $570 \mathrm{~nm}$ using a standard spectrophotometer.

Immunofluorescence. For immunofluorescence experiments, cells were fixed in $4 \%$ paraformaldehyde directly added to culture medium for $10 \mathrm{~min}$, then washed in PBS two times, treated with $0.1 \mathrm{M}$ glycine for $4 \mathrm{~min}$ in PBS and permeabilized with $0.1 \%$ Triton X-100 in PBS for another 4 min. After washing with PBS and blocking with $0.2 \%$ BSA, $1 \%$ NGS, $0.1 \%$ Triton X-100 in PBS (blocking solution), cells were incubated with the indicated antibodies diluted in blocking solution for $90 \mathrm{~min}$ at room temperature. We used anti-FLAG (1:1000, Sigma Aldrich), anti-TTRAP (1:100), anti-NPM (1:100, Invitrogen), anti-Fibrillarin (38F3; 1:100, Santa Cruz Biotechnology, Santa Cruz, CA, USA), anti-NCL (1:100, Invitrogen), anti-UBF (F-9; 1:100, Santa Cruz Biotechnology), anti-PML (PG-M3; 1:100, Santa Cruz Biotechnology), anti-DAXX (1:100, Santa Cruz Biotechnology), anti-FLASH (M-300; 1:100, Santa Cruz Biotechnology), anti-Coilin (ab11822; 1:100, Abcam, Cambridge, UK), anti-SC35 (1:300, Sigma Aldrich), anti-20S proteasome (1:200, Biomol, Enzo Life Sciences, Farmingdale, NY, USA), antiUbiquitin (1: 100, DakoCytomation, Glostrup, Denmark), anti-p53 (D01, Santa Cruz Biotechnology), anti gamma-H2AX (1:100, Upstate, Charlottesville, VA, USA) anti-ATM (1:100, Rockland, Gilbertsville, PA, USA), anti-Mre11 (1:100, Novus, Littleton, CO, USA). For detection, cells were incubated with Alexa Fluor-488 or -594 (Molecular Probes, Invitrogen) labeled anti-mouse or anti-rabbit secondary antibodies. For nuclear staining, cells were incubated with DAPI $(1 \mu \mathrm{g} / \mathrm{ml})$ for $5 \mathrm{~min}$.

Triple immunofluorescence was performed using Zenon technology: anti-NPM antibody was labeled with Zenon Alexa Fluor 405 Mouse $\operatorname{lgG}^{1}$ labeling reagent (Invitrogen), according to manufacturer's instructions.

Cells were washed and mounted with Vectashield mounting medium (Vector, Burlingame, CA, USA). All images were collected using a confocal laser scanning microscope LEICA TCS SP2 (Leica Microsystems GmbH, Wetzlar, Germany). In double and triple staining, confocal sections of fluorescent signals were taken using the sequential mode (between lines) and merged images of the two/three signals were obtained using confocal microscope software.

Confocal laser scanning microscopy. All images were collected using a confocal laser scanning microscopy LEICA TCS SP2 supplemented with an Argon/ Krypton laser, an Helium/Neon laser, a 405-nm laser and equipped with $63 \times 1.4$ oil immersion lenses. The excitation/emission wavelengths employed were $488 \mathrm{~nm} /$ $519 \mathrm{~nm}$ for Alexa Fluor 488 labeling, $594 \mathrm{~nm} / 617 \mathrm{~nm}$ for Alexa Fluor 594 labeling and $405 \mathrm{~nm} / 421 \mathrm{~nm}$ for Alexa Fluor 405 labeling. In double and triple staining, confocal sections of fluorescent signals were taken using the sequential mode (between lines) and merged images of the two signals were obtained using confocal microscope software. For 3D reconstruction and z-axis projection analysis, confocal sections were taken at intervals of $0.3 \mu \mathrm{m}$ inside cells. For colocalization experiments, high-resolution images were analyzed with ImageJ software (http:// rsbweb.nih.gov/ij/index.html) using intensity correlation analysis plug-in.

Immunoprecipitation and western blot analysis. For coimmunoprecipitation experiments, cells were lysed in PML lysis buffer $(150 \mathrm{mM}$ $\mathrm{NaCl}, 50 \mathrm{mM}$ Tris (pH 7.5), $1 \% \mathrm{NP} 40$ ), supplemented with protease inhibitor cocktail (Roche, Basel, Switzerland) and with $20 \mathrm{mM} \mathrm{N}$-ethylmaleimide. Cellular lysates were incubated with anti-FLAG agarose beads (Sigma Aldrich) or with anti-PML antibody. Mouse IgGs were used in negative control reactions. After washing, immunoprecipitated proteins were eluted with $2 \times$ SDS sample buffer, boiled and analyzed by western blot. The following antibodies were used: anti-FLAG 1:2000 (Sigma), anti-MYC 1:4000 (Cell Signaling, Beverly, MA, USA), anti-TTRAP $1: 1000,{ }^{8}$ anti-PML $1: 1000$ (Santa Cruz).

Biochemical separation of nuclear and cytoplasmic fractions was done as previously described, without RNAse inhibitor. ${ }^{37}$ Separated fractions were analyzed by western blot with anti-TTRAP and anti-retinoblastoma (1:1000, BD Biosciences, Franklin Lakes, NJ, USA) antibodies. Anti- $\beta$-actin $(1: 5000)$ was used as loading control. 
Constructs. pCDNA3-2XFLAG-TTRAP and pCS2-6XMYC-TTRAP were previously described. ${ }^{8}$ FLAG-TTRAP-SIM mutant was generated by PCR as in Hecker et al. ${ }^{6}$ Plasmids encoding $5^{\prime}$-phospodiestarase inactive TTRAP mutants E152A and D262A were kindly provided by Dr. KW Caldecott (University of Sussex, Brighton, UK). pCDNA3-PML-IV and pSG5-PML-RARá constructs have been previously described. ${ }^{38}$ Two different oligonucleotide sequences were selected for silencing PML expression using siRNA Target Finder software (Invitrogen). The hairpin-encoding oligonucleotides were cloned into the pSuperior vector (Invitrogen). The following oligonucleotide sequences were used: siPML\#1 (5'-CGGAGGAGGAGTTCCAGTTT-3'), siPML\#2 (5'-CCCCGCAAGACCAACAAC AT-3'), scramble ( $5^{\prime}$-CACAGTCGGTTTGGAGTGAG-3'; Invitrogen).

RNA isolation, reverse transcription and qPCR. Total RNA was isolated using the TRIZOL reagent (Invitrogen) following the manufacturer's instructions. Single strand cDNA was obtained from $1 \mu \mathrm{g}$ of purified RNA using the iSCRIPT cDNA Synhesis Kit (Bio-Rad, Hercules, CA, USA) according to manufacturer's instructions. qPCR was performed using SYBR-Green PCR Master Mix (Applied Biosystem, Foster City, CA, USA) and an iCycler IQ Real time PCR System (Bio-Rad). Primers for $A 0,{ }^{17} \beta$-actin and $\mathrm{GAPDH}^{39}$ were previously described. Oligonucleotides for 1 and 4 cleavage sites were designed on human reference sequences as following: for cleavage site 1, FWD $5^{\prime}$-CGCTC TACCTTACCTACCTG-3' and REV 5'- CTAGAATTACCACAGTTATCCAAG-3'; and for cleavage site 4, FWD $5^{\prime}$-TAGAGGAAGTAAAAGTCGTAAC- $3^{\prime}$ and REV 5'-GGTGGGTGTGCGGAGGGAAG-3'.

${ }^{32} \mathrm{P}$-orthophosphate in vivo labeling and RNA analysis. Metabolic labeling and analysis of rRNA was carried out as previously described. ${ }^{26}$ Briefly, siTTRAP and scramble control cells were treated with $5 \mu \mathrm{M}$ MG132 for $16 \mathrm{~h}$ or left untreated. Drug was maintained in the medium during the entire period of the procedure. Cells were incubated with phosphate-free medium supplemented with $10 \%$ dialyzed FBS (labeling medium) for $1 \mathrm{~h}$ before labeling with ${ }^{32} \mathrm{P}$-orthophosphate $(15 \mu \mathrm{Ci} / \mathrm{ml})$ in labeling medium for $1 \mathrm{~h}$ (pulse). Medium was then replaced with complete medium for $1 \mathrm{~h}$ or $3 \mathrm{~h}$ (chase). In all, $5 \mu \mathrm{M}$ MG132 was maintained in the medium during the entire procedure. Total RNA was extracted using TRIZOL reagent (Invitrogen) and $1 \mu \mathrm{g}$ of purified RNA was separated on $1 \%$ agarose-formaldehyde gel. After electrophoresis, 28S and 18S rRNA were controlled under UV light and gels were dried. rRNA species were detected by autoradiography.

Statistical analysis. All experiments were repeated in triplicate or more. For stably transfected cells, at least two independent clones were used for each cell line in all experiments. Data represent the mean with standard deviation. When necessary, each group was compared individually with reference control group using Student's t-test (Microsoft Excel software).

\section{Conflict of Interest}

The authors declare no conflict of interest.

Acknowledgements. We are indebted to all the members of the SG lab for thought-provoking discussions and to Cristina Leonesi for technical support. We thank KW Caldecott (University of Sussex, Brighton, UK) for mutant TTRAP E152A and D262A constructs, V De Laurenzi (University of Rome Tor Vergata, Rome, Italy) for anti-FLASH and anti-coilin antibodies, and E Buratti (ICGEB, Trieste, Italy) for anti-SC35 antibody. We also thank Rosanna Parlato for helpful discussions and sharing of unpublished data. We would also like to thank Micaela Grandolfo for technical support with confocal microscope and Silvano Piazza for insights into image analysis softwares. This work was supported by the Telethon Grant GGP06268, by the Giovanni Armenise-Harvard Foundation and by the Italian Institute of Technology.

1. Cortes Ledesma F, El Khamisy SF, Zuma MC, Osborn K, Caldecott KW. A human 5'tyrosyl DNA phosphodiesterase that repairs topoisomerase-mediated DNA damage. Nature 2009; 461: 674-678.

2. Rodrigues-Lima $F$, Josephs $M$, Katan M, Cassinat B. Sequence analysis identifies TTRAP, a protein that associates with CD40 and TNF receptor-associated factors, as a member of a superfamily of divalent cation-dependent phosphodiesterases. Biochem Biophys Res Commun 2001; 285: 1274-1279.
3. Pype S, Declercq W, Ibrahimi A, Michiels C, Van Rietschoten JG, Dewulf N et al. TTRAP, a novel protein that associates with CD40, tumor necrosis factor (TNF) receptor-75 and TNF receptor-associated factors (TRAFs), and that inhibits nuclear factor-kappa $B$ activation. J Biol Chem 2000; 275: 18586-18593.

4. Pei $H$, Yordy JS, Leng Q, Zhao Q, Watson DK, Li R. EAPII interacts with ETS1 and modulates its transcriptional function. Oncogene 2003; 22: 2699-2709.

5. Esguerra CV, Nelles L, Vermeire L, Ibrahimi A, Crawford AD, Derua R et al. Trap is an essential modulator of Smad3-dependent Nodal signaling during zebrafish gastrulation and left-right axis determination. Development 2007; 134: 4381-4393.

6. Hecker C, Rabiller M, Haglund K, Bayer P, Dikic I. Specification of SUMO1- and SUMO2interacting motifs. J Biol Chem 2006; 281: 16117-16127.

7. Xu G, Pan Y, Wang B, Huang L, Tian L, Xue J et al. TTRAP is a novel PML nuclear bodiesassociated protein. Biochem Biophys Res Commun 2008; 375: 395-398.

8. Zucchelli S, Vilotti S, Calligaris R, Lavina ZS, Biagioli M, Foti R et al. Aggresome-forming TTRAP mediates pro-apoptotic properties of Parkinson's disease-associated DJ-1 missense mutations. Cell Death Differ 2009; 16: 428-438.

9. Li C, Fan S, Owonikoko TK, Khuri FR, Sun S, Li R. Oncogenic role of EAPII in lung cancer development and its activation of the MAPK-ERK pathway. Oncogne 2011; 30: 3802-3812.

10. Lee HY, Youn SW, Kim JY, Park KW, Hwang Cl, Park WY et al. FOXO3a turns the tumor necrosis factor receptor signaling towards apoptosis through reciprocal regulation of c-Jun $\mathrm{N}$-terminal kinase and NF-kappaB. Arterioscler Thromb Vasc Biol 2008; 1: 112-120.

11. Mattsson K, Pokrovskaja K, Kiss C, Klein G, Szekely L. Proteins associated with the promyelocytic leukemia gene product (PML)-containing nuclear body move to the nucleolus upon inhibition of proteasome-dependent protein degradation. Proc Natl Acad Sci USA 2001; 98: 1012-1017

12. Karni-Schmidt O, Zupnick A, Castillo M, Ahmed A, Matos T, Bouvet $P$ et al. p53 is localized to a sub-nucleolar compartment after proteasomal inhibition in an energy-dependent manner. J Cell Sci 2008; 121: 4098-4105.

13. Krüger $T$, Scheer U. p53 localizes to intranucleolar regions distinct from the ribosome production compartments. J Cell Sci 2010; 123: 1203-1208.

14. Shen TH, Lin H, Scaglioni PP, Yung TM, Pandolfi PP. The mechanisms of PML-nuclear body formation. Mol Cell 2006; 24: 331-339.

15. Fu C, Ahmed K, Ding H, Ding X, Lan J, Yang Z et al. Stabilization of PML nuclear localization by conjugation and oligomerization of SUMO-3. Oncogene 2005; 24: 5401-5413.

16. Perry RP, Kelley DE. Inhibition of RNA synthesis by actinomycin D: characteristic doseresponse of different RNA species. J Cell Physiol 1970; 76: 127-139.

17. Murayama A, Ohmori K, Fujimura A, Minami H, Yasuzawa-Tanaka K, Kuroda T et al. Epigenetic control of rDNA loci in response to intracellular energy status. Cell 2008; 133: 627-639.

18. Henras AK, Soudet J, Gérus M, Lebaron S, Caizergues-Ferrer M, Mougin A et al. The post-transcriptional steps of eukaryotic ribosome biogenesis. Cell Mol Life Sci 2008; 65 : 2334-2359.

19. Dez C, Houseley J, Tollervey D. Surveillance of nuclear-restricted pre-ribosomes within a subnucleolar region of Saccharomyces cerevisiae. EMBO J 2006; 25: 1534-1546.

20. LaRiviere FJ, Cole SE, Ferullo DJ, Moore MJ. A late-acting quality control process for mature eukaryotic rRNAs. Mol Cell 2006; 24: 619-626.

21. Blaise R, Masdehors $\mathrm{P}$, Laugé $\mathrm{A}$, Stoppa-Lyonnet $\mathrm{D}$, Alapetite $\mathrm{C}$, Merle-Béral $\mathrm{H}$ et al. Chromosomal DNA and p53 stability, ubiquitin system and apoptosis in B-CLL lymphocytes. Leuk Lymphoma 2001; 42: 1173-1180.

22. Stavreva DA, Kawasaki M, Dundr M, Koberna K, Müller WG, Tsujimura-Takahashi T et al. Potential roles for ubiquitin and the proteasome during ribosome biogenesis. $\mathrm{Mol} \mathrm{Cell} \mathrm{BiO}$ 2006; 26: 5131-5145.

23. Campagnoli MF, Ramenghi U, Armiraglio M, Quarello P, Garelli E, Carando A et al. RPS19 mutations in patients with Diamond-Blackfan anemia. Hum Mutat 2008; 29: 911-920.

24. Narla A, Ebert BL. Ribosomopathies: human disorders of ribosome dysfunction. Blood 2010; 115: 3196-3205.

25. Ruggero D, Pandolfi PP. Does the ribosome translate cancer? Nat Rev Cancer 2003; 3 : 179-192.

26. Burger K, Mühl B, Harasim T, Rohrmoser M, Malamoussi A, Orban M et al. Chemotherapeutic drugs inhibit ribosome biogenesis at various levels. J Biol Chem 2010; 285: 12416-12425.

27. Grandori C, Gomez-Roman N, Felton-Edkins ZA, Ngouenet C, Galloway DA, Eisenman RN et al. c-Myc binds to human ribosomal DNA and stimulates transcription of rRNA genes by RNA polymerase I. Nat Cell Biol 2005; 7: 311-318.

28. Robledo S, Idol RA, Crimmins DL, Ladenson JH, Mason PJ, Bessler M. The role of human ribosomal proteins in the maturation of rRNA and ribosome production. RNA 2008; 14: 1918-1929.

29. Finkbeiner $E$, Haindl M, Muller $S$. The SUMO system controls nucleolar partitioning of a novel mammalian ribosome biogenesis complex. EMBO J 2011; 30: 1067-1078.

30. Pontes O, Li CF, Nunes PC, Haag J, Ream T, Vitins A et al. The Arabidopsis chromatinmodifying nuclear siRNA pathway involves a nucleolar RNA processing center. Cell 2006; 126: 79-92.

31. Lebreton A, Tomecki R, Dziembowski A, Séraphin B. Endonucleolytic RNA cleavage by a eukaryotic exosome. Nature 2008; 456: 993-996. 
32. Allmang C, Kufel J, Chanfreau G, Mitchell P, Petfalski E, Tollervey D. Functions of the exosome in rRNA, snoRNA and snRNA synthesis. EMBO J 1999; 18: 5399-5410.

33. Zhou Y, Schmitz K, Mayer C, Yuan X, Akhtar A, Grummt I. Reversible acetylation of the chromatin remodelling complex NoRC is required for non-coding RNA-dependent silencing. Nat Cell Biol 2009; 11: 1010-1016.

34. Tanaka Y, Okamoto K, Teye K, Umata T, Yamagiwa N, Suto Y et al. JmiC enzyme KDM2A is a regulator of rRNA transcription in response to starvation. EMBO J 2010; 29: 1510-1522.

35. Andersen JS, Lam YW, Leung AKL, Ong S, Lyon CE, Lamond Al et al. Nucleolar proteome dynamics. Nature 2005; 433: 77-83.

36. McMahon M, Ayllón V, Panov KI, O'Connor R. Ribosomal 18 S RNA processing by the IGF-I-responsive WDR3 protein is integrated with p53 function in cancer cell proliferation J Biol Chem 2010; 285: 18309-18318.
37. Wang Y, Zhu W, Levy DE. Nuclear and cytoplasmic mRNA quantification by SYBR green based real-time RT-PCR. Methods 2006; 39: 356-362.

38. Fogal V, Gostissa M, Sandy P, Zacchi P, Sternsdorf T, Jensen K et al. Regulation of p53 activity in nuclear bodies by a specific PML isoform. EMBO J 2000; 19 : 6185-6195.

39. Zucchelli S, Codrich M, Marcuzzi F, Pinto M, Vilotti S, Biagioli M et al. TRAF6 promotes atypical ubiquitination of mutant DJ-1 and alpha-synuclein and is localized to Lewy bodies in sporadic Parkinson's disease brains. Hum Mol Genet 2010; 19: 3759-3770.

40. Morello LG, Hesling C, Coltri PP, Castilho BA, Rimokh R, Zanchin NIT. The NIP7 protein is required for accurate pre-rRNA processing in human cells. Nucleic Acids Res 2010; 39 648-665.

Supplementary Information accompanies the paper on Cell Death and Differentiation website (http://www.nature.com/cdd) 